\title{
Student mental models about conductors and dielectrics
}

\author{
Jing Zhang ${ }^{1}$ and Lin Ding ${ }^{1,2}$ \\ ${ }^{1}$ School of Physics and Optoelectronic Engineering, Yangtze University, Hubei 434023, People's Republic of China \\ ${ }^{2}$ Department of Teaching and Learning, The Ohio State University, Columbus, Ohio 43210, USA
}

\begin{abstract}
We classified students' mental models on conductors and dielectrics into four levels that vary progressively from naïve views to scientifically acceptable views, namely non-model, non-scientific model, flawed-scientific model, and scientific model. A total of 137 Chinese students from an introductory physics class completed a questionnaire that targets learners' conceptual understanding of conductors and dielectrics. For the questions on conductors, about $20 \%$ of the students were at the non-model level, and almost $15 \%$ of the students constructed a non-scientific model and believed that both electrons and protons could move in a conductor. Those with a flawed-scientific model (35\%) were unsure about the motion of the particles. Only 30\% of the students had a scientific model. For the dielectrics questions, over $30 \%$ of the students were in the non-model level; about $55 \%$ of the students had a non-scientific model and thought that charges could not move in a dielectric. The remaining $10 \%$ of the students used a flawed-scientific model.
\end{abstract}

\section{INTRODUCTION}

One of the theories about knowledge organization is "mental model." There has been an increased consideration of the role of mental models in science education. According to the new framework for k-12 science education, model as a crosscutting concept can be divided into conceptual model (external) and mental model (internal) [1]. In the existing literature, there are various definitions of mental models. For example, Vosniadou explained that mental models "refer to a special kind of mental representation, an analog representation, which individuals generate during cognitive functioning." [2]. Greca and Moreira defined "mental model as an internal representation, which acts out as a structural analogue of situations or processes. Its role is to account for the individuals' reasoning both when they try to understand discourse and when they try to explain and predict the physical world behavior." [3]. Hrepic, Zollman, and Rebello considered mental models as "a mental structure built of more fundamental cognitive and knowledge elements" [4] and stressed "coherent" organization of these elements in forming a mental model [5]. Drawing on these studies, we use the term "mental model" to describe the dynamic cognitive structure that is built on more fundamental cognitive and knowledge elements and is created on the spot for the purpose of answering questions, solving problems, or dealing with other situations.

In China, physics teaching has long been focusing on external conceptual models of abstract topics presented by textbook writers and teachers. However, less attention is given to students' mental models that are constructed by the students themselves. Research in science education increasingly advocates the use of a learning progression approach to describe and interpret how students develop their understandings of a given concept such as energy. That said, studies concerning the learning progression of mental models are rather scant.

Conductors and dielectrics are commonly used in everyday life. Students often have many non-scientific models about these concepts. Therefore, students' mental models revealed in this study can be useful empirical sources for designing teaching strategies. Specifically, this study is designed to answer the following research question: How can we identify and describe the progression of introductory physics students' mental models about conductors and dielectrics under different contexts?

\section{THEORETICAL FRAMEWORK}

Learning progression, as a research method, is used to systematically investigate students' science learning processes in order to build a bridge between research on learning and instructional practices [6]. It also provides a framework for bringing coherence to multiple facets of the educational system including standards, curricula, assessments, and teacher professional development [7].

In the existing research, different progression variables have been studied, such as content knowledge as a variable (like energy) or practice as a variable (like modeling, argumentation and so on). Here we choose mental model as a progression variable and classify the levels of student models vary progressively from naïve views to scientifically acceptable views.

A mental model is built on fundamental cognitive and knowledge elements as well as on their relationships, which, when assembled into a mental model become its features [2]. Elements are divided into key elements and other 


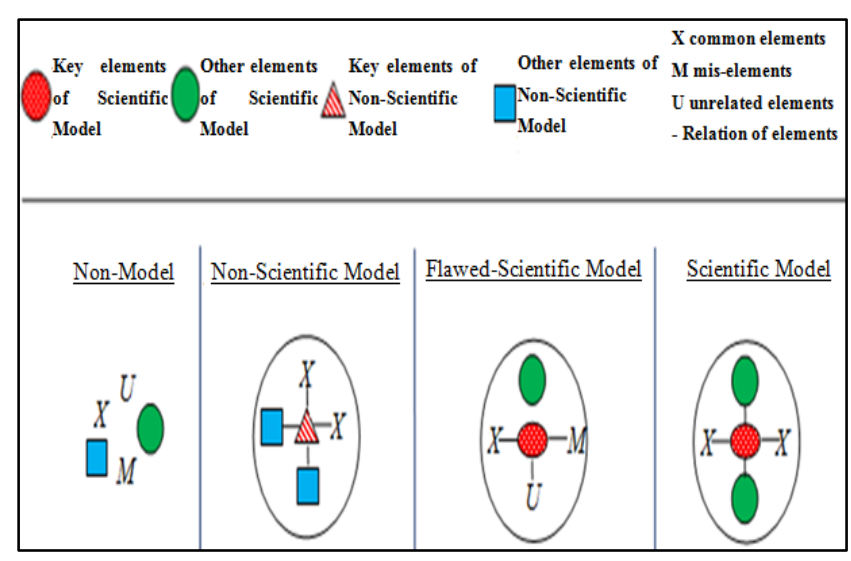

FIG.1. The framework of progression of mental models.

elements. Key elements are the most important and are core concepts or descriptions of the mental model. Comparatively, other elements can be considered as auxiliary ideas. Students' levels are defined by the mental models that they use across different contexts.

Based on the mental model theories and learning progression studies [4, 8-9], our framework of progression of mental models is depicted in FIG.1. Students' mental models are classified into four levels: non-model, non-scientific model, flawed-scientific model, and scientific model. Students who use disconnected knowledge elements with no attempt to make connections are at the "non-model" level. Students in the "non-scientific model" level construct a model with non-scientific knowledge elements, and therefore their models are incomplete and non-scientific. Students in the "flawed-scientific model" level use key scientific knowledge elements but have some flaws in other elements. For instance, students may present some unrelated elements or establish incorrect relationships between other knowledge elements. The "scientific model" means that the students' mental models are both complete and scientific.

\section{METHODOLOGY AND RESEARCH DESIGN}

Participants in this study were 137 sophomore telecommunication majors taking an introductory physics class at a 4-year university in China. Since we had no way to "see" the participants' mental models directly, we could only attempt to infer their mental models through their expressed responses, which was the information conveyed through their verbal or other expressive means[10], such as pictures or graphs. We used 6 open-ended questions to elicit their mental models; three on conductors and three on dielectrics with each being framed in a different context, such as direct contact, rubbing and induction (see FIG.2). These questions were adopted from the existing literature $[11,12]$. Students were given 25 minutes to complete these

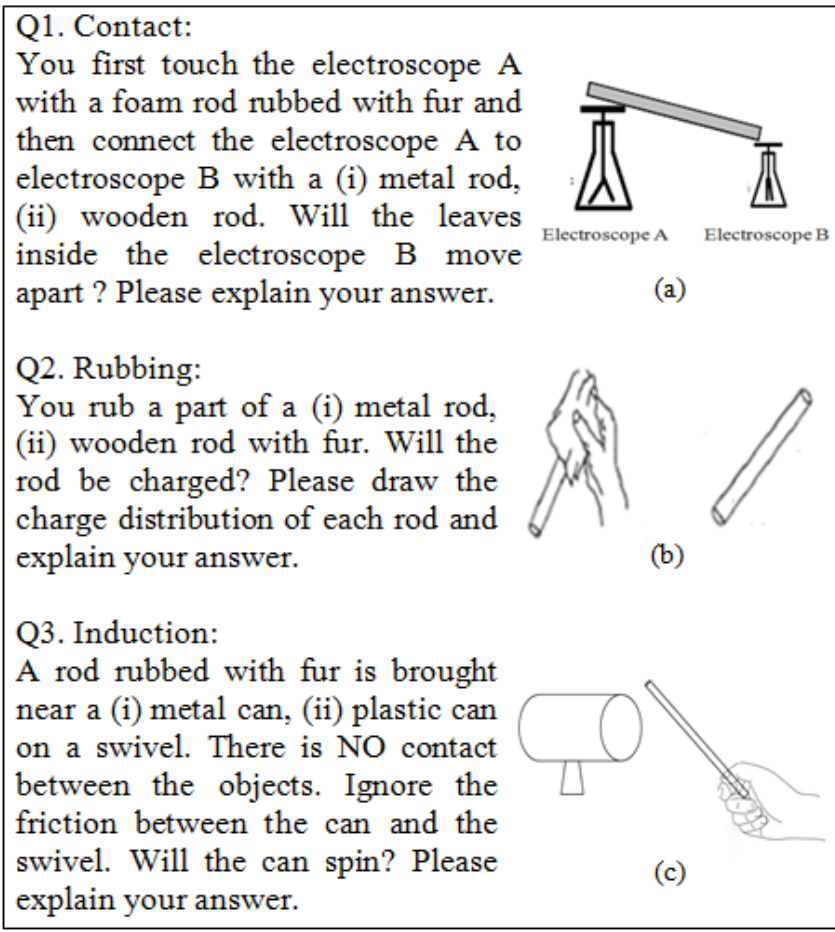

FIG.2. Questions for eliciting students' mental models about conductors and dielectrics.

questions. They were required to predict the experiment results and to use words and figures to give detailed explanations.

We first developed a progression structure (with the above-mentioned four levels) for the topics of conductors and dielectrics based on the pertinent literature on students' misconceptions [11, 13]. Next, we examined the students' responses using our proposed levels and at the same time revised the progression structure to develop detailed descriptions for each level. After several iterations, a final version of the mental model progression levels was completed (see Table I and Table II). Using the progression structure, two researchers independently classified students' responses of each context into different levels. This resulted in an inter-reliability rate of $89 \%$. All remaining differences were resolved after discussion between the researchers.

TABLE I. The Progression structure of conductors

\begin{tabular}{ll}
\hline \multicolumn{1}{c}{ Level } & Feature \\
\hline Non- model & $\begin{array}{l}\text { No model-like explanation or no } \\
\text { answer. }\end{array}$ \\
\hline Non-scientific & $\begin{array}{l}\text { Electrons and protons all can move } \\
\text { freely. }\end{array}$ \\
\hline Flawed-scientific & $\begin{array}{l}\text { Electrons can move freely, but } \\
\text { students were unsure about the } \\
\text { motion of the protons. }\end{array}$ \\
\hline Scientific & Only electrons can move freely. \\
\hline
\end{tabular}


TABLE II. The Progression structure of dielectrics.

\begin{tabular}{ll}
\hline \multicolumn{1}{c}{ Level } & \multicolumn{1}{c}{ Feature } \\
\hline Non-model & $\begin{array}{l}\text { No model-like explanations or no } \\
\text { answer. }\end{array}$ \\
\hline Non-scientific & $\begin{array}{l}\text { Charges cannot move at all or can } \\
\text { move freely in dielectrics. }\end{array}$ \\
\hline Flawed-scientific & $\begin{array}{l}\text { Electrons cannot move freely in } \\
\text { dielectrics. However, the student } \\
\text { does not know the mechanisms of } \\
\text { induction. }\end{array}$ \\
\hline
\end{tabular}

Electrons cannot move freely in

dielectrics. When a rod is rubbed,

Scientific electrons can move from one material to another. Electrons can shift slightly when polarized.

*The bold prints are key elements of a scientific model.

\section{RESULTS}

For conductors, it was found that about $20 \%$ of the students were at the non-model level (see Table III). They either did not provide sensible model-like explanations or provided no answer at all. Nearly $15 \%$ of the students constructed a non-scientific model and believed that both electrons and protons could move freely in a conductor. An example of a student's drawing of this kind of model under the context of Q1(i) is reproduced in FIG.3 (a). Those with a flawed-scientific model (about 30\%) knew that electrons could move freely but were unsure about the motion of the protons. They guessed that the protons could move freely or could not move at all, which suggested that they didn't know the exact electronic model of conductors. Only 30\% of the students had a scientific model, and they understood that only electrons could move freely. We also found the percentages of each level were slightly different under different context for Q1(i), Q2(i) and Q3(i). Especially for Q2(i), most students who were able to predict the correct phenomenon for Q1(i) and Q3(i) found it difficult to make a prediction for Q2(i), and even more so to explain it. Compared with the other two questions, the context of Q2(i) [c.f. FIG.2 (b)] was an unfamiliar context for most students, because the scenario of static charges induced by rubbing only occurred in non-conductors. Students didn't know why this method would not work for charging conductor. They didn't consider that the charges could easily move from the

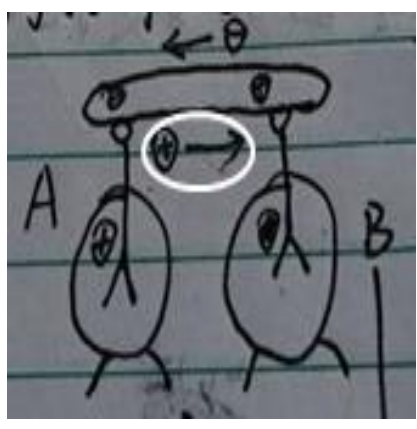

(a)Q1(i)

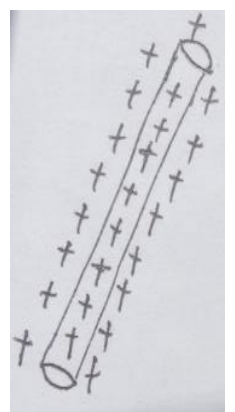

(b)Q2(ii)
FIG.3. Examples of students' drawing of the non-scientific model of conductors and dielectrics

metal rod to the person who in principal was a conductor. Instead, the students thought the charges inside the conductors did not move. The number of the students with such a non-scientific model increased by almost $15 \%$ compared the other two contexts.

In the case of dielectrics questions, about $30 \%$ of the students were in the non-model level; more than $40 \%$ of the students had a non-scientific model and thought that charges could not move at all or could move freely in dielectrics. Almost $10 \%$ of the students used a flawed-scientific model, in which case, they knew that electrons could not move freely in dielectrics but did not know the mechanisms of polarization. Only about 5\% had a scientific model. Interestingly, the number of the students who exhibited a flawed-scientific model in answering Q2(ii) was higher than in Q1(ii) and Q3(ii). As mentioned above, the context of rubbing a non-conductor was familiar to students. They all knew dielectrics could gain or lose electrons, but they thought the electrons could not move through direct contact and induction as depicted in Q1(ii) and Q3(ii). Specifically many students showed a lack of understanding of dielectric polarization. So, for Q1(ii), when a wooden rod was placed between a charged electroscope and a neutral electroscope, as shown in FIG. 2(a), many students thought that "the leaves inside the electroscope B will not move apart". As for Q3(ii), the majority of the students made an incorrect prediction that the plastic can would remain motionless on a swivel when a rubbed rod was brought near. Here, the wooden rod and the

TABLE III. Students' mental model of conductors and dielectrics $(\mathrm{n}=137)$

\begin{tabular}{lllllll}
\hline \multirow{2}{*}{ Level } & \multicolumn{3}{c}{ Conductors } & \multicolumn{3}{c}{ Dielectrics } \\
\cline { 2 - 7 } & Q1(i) & Q2(i) & Q3(i) & Q1(ii) & Q2(ii) & Q3(ii) \\
\hline Non-model & $21 \%$ & $18 \%$ & $18 \%$ & $25 \%$ & $30 \%$ & $28 \%$ \\
\hline Non-scientific & $14 \%$ & $33 \%$ & $17 \%$ & $59 \%$ & $42 \%$ & $56 \%$ \\
\hline Flawed-scientific & $33 \%$ & $28 \%$ & $34 \%$ & $9 \%$ & $20 \%$ & $11 \%$ \\
\hline Scientific & $32 \%$ & $21 \%$ & $31 \%$ & $7 \%$ & $8 \%$ & $5 \%$ \\
\hline
\end{tabular}


plastic can are non-conductors, which means that the electrons therein are not mobile and will remain bound. However, when a charged material is brought near to them, the electric field of the charged material will repel or attract the electron clouds surrounding the atoms inside the wooden rod or the plastic can. As a result, they will be polarized. Regarding Q2, although students knew about free-moving electrons by rubbing as was the case in Q2(ii),almost 30\% of them drew the charges as being distributed on the whole rod like FIG.3(b). In other words, the students had a non-scientific model and thought charges could move freely in dielectrics. In fact, the charges produced by rubbing stay localized.

\section{CONCLUSIONS AND IMPLICATIONS}

In this study, we analyzed students' predictions and explanations about conductors and dielectrics under the contexts of direct contact, rubbing and induction. First, the framework of the mental model progression helped us identify and distinguish the various levels of students' mental models. The progression structure of the students' mental models about conductors and dielectrics was manifested in their responses to the different questions. Second, we found almost over $15 \%$ of the students held the non-scientific model of conductors and about $30 \%$ students had a flawed-scientific model. They were not sure whether the protons could move when there was an electric field. For the dielectrics questions, over $40 \%$ of the students had a non-scientific model, and only about $5 \%$ had a scientific model. Most students showed a lack of understanding of polarization, even though they were familiar with situations such as a rubbed rod attracting small pieces of paper. This particular outcome is consistent with those reported in the existing literature [11]. Further, the student distributions across the levels varied under different contexts. Students' mental models were somewhat sensitive to unfamiliar contexts. Students were not familiar with the situation of rubbing conductors and polarization in dielectrics. When they dealt with questions that they hadn't seen before, for example, Q2(i), or when the situation was modified from their familiar contexts, such as Q3(ii), the percentages of non-scientific model tended to increase. Conversely, in familiar contexts, such as Q1(i) and Q3(i), students' mental models across the different situations seemed to remain relatively stable.

These results have important implications for teaching. It is important that we use multiple teaching strategies to develop students' mental models. When most students hold non-scientific models, we may consider using cognitive conflict strategies to stimulate a desired conceptual change. For example, in order to change students' non-scientific model of dielectrics that charges cannot move in a dielectric, creating cognitive dissonances among students by asking them to make predictions before experimentation can be helpful. If most students have flawed-scientific models, perhaps using bridging strategies that draw on students' existing mental models to help them reach the scientific level will be useful. In short, we need to provide students opportunities to use their mental models under different contexts to make their concept application more flexible.

\section{ACKNOWLEDGMENTS}

This study is supported by the National Science Foundation (\#1252399), China College Physics Teaching Committee (\#DWJZW201534zn), and Department of Education of Hubei Province (\#2015270).
[1] NRC. A framework for K-12 science education: practices, crosscutting concepts, and core ideas. (National Research Council, Washington, 2011), p.117.

[2] S. Vosniadou, Capturing and modeling the process of conceptual change, Learn. Instr. 4, 45 (1994).

[3] I. M. Greca and M. A. Moreira, Mental, physical and mathematical models in the teaching and learning of physics, Sci. Educ. 86, 106 (2002).

[4] Z. Hrepic, D. A. Zollman, and N. S. Rebello, Identifying students' mental models of sound propagation: The role of conceptual blending in understanding conceptual change, Phys. Rev. ST Phys. Educ. Res. 6, 020114 (2010).

[5] Nilüfer Didiş, Ali Eryilmaz, and Şakir Erkoç, Investigating students' mental models about the quantization of light, energy and angular momentum, Phys. Rev. ST Phys. Educ. Res. 10, 020127 (2014).

[6] Salinas, I. Learning progressions in science education: Two approaches for development. In Learning Progressions in Science (LeaPS) Conference, Iowa City, IA. . (2009)

[7] A. C. Alonzo, \& A. W. Gotwals. Learning progressions in science: Current challenges and future directions. (Sense publishers ,Rotterdam , 2012), p.3

[8] S.Y. Stevens, C. Delgado, J. S. Krajcik: Hypothetical Multi-Dimensional Learning Progression, JRST. 47, 687(2010).

[9] S. Vosniadou and W. F. Brewer, Mental models of the earth: A study of conceptual change in childhood, Cogn.Psychol. 24, 535 (1992).

[10] D. Norman, Some observations on mental models, in Mental Models, edited by D. Gentner and A. Stevens (Lawrence Erlbaum Associates, Hillsdale, NJ, 1983).

[11] Jongwon Park, Kwangju, Ikgyun, Kim,Chongju, Myunghwan Kim and Moo Lee. Analysis of students' processes of confirmation and falsification of their prior ideas about electrostatics. Int.J.Sci.Educ , 23,1219 (2001).

[12]http://paer.rutgers.edu/pt3/experimentindex.php?topicid $=10 \&$ cycleid $=50$. retrieved 4/24/2012

[13] C. Guruswamy, M. D. Somers \& R. G. Hussey.. Students' understanding of the transfer of charges between conductors .Phys. Educ, 32,91(1997). 